\title{
Falha de extubação e suas implicações clínicas em unidade de terapia intensiva
}

\author{
Juliana Harumi Hattori Sauragi Kavaturo', Fernando Osni Machado², Leonardo Jönck Staub ${ }^{2}$, Rosemeri \\ Maurici da Silva
}

1. Especialista, Mestrado Profissional em Cuidados Intensivos e Paliativos, Universidade Federal de Santa Catarina, Florianópolis, SC.

2. Doutor, Departamento de Clínica Médica, Universidade Federal de Santa Catarina, Florianópolis, SC.

3. Doutora, Programa de Pós-Graduação em Ciências Médicas, Universidade Federal de Santa Catarina, Florianópolis, SC.

\section{RESUMO}

Introdução. $\mathrm{O}$ uso da ventilação mecânica (VM) aumentou significativamente nas últimas décadas e atualmente é uma importante modalidade terapêutica. Contudo, está associada a complicações que colocam em risco os cuidados do paciente crítico, tornando um grande desafio a identificação do momento correto para extubar um paciente, buscando-se evitar tanto um prolongamento desnecessário da VM como uma extubação prematura, pois ambos estão relacionados ao aumento do risco de complicações. Objetivos. Identificar os fatores associados à falha de extubação, buscando a caracterização de pacientes com risco do seu insucesso e avaliar as implicações clínicas relacionadas com a necessidade de reintubação. Métodos. Foi realizado um estudo de coorte incluindo pacientes internados na unidade de terapia intensiva (UTI) submetidos à VM. Esses foram acompanhados até 48 horas após a extubação, permanecendo aqueles que apresentaram o desfecho considerado neste estudo, como reintubação dentro de 48 horas após a extubação ou a evolução para o óbito durante esse período. Resultados. 83 pacientes foram elegíveis para este estudo. Houve associação significante entre o maior tempo de UTI (p 0,048), maior tempo de VM (p 0,040) e mortalidade na UTI (p 0,022) com falha de extubação. Foram também observados elevação de complicações associadas ao uso do suporte ventilatório, como pneumonia associada à VM (p 0,001) e frequência de traqueostomia (p 0,009). Conclusão. A falha de extubação está associada a aumento da mortalidade na UTI, do tempo de permanência, e duração na ventilação mecânica.

DOI: https://doi.org/10.32963/bcmufsc.v6i1.3927

Palavras-chave: Extubação; Desmame do Respirador; Respiração Artificial. Submetido em 26/02/2020; aceito para publicação em 23/05/2020.

Os autores declaram não possuir conflitos de interesse.

Autor para contato: Profa. Dra. Rosemeri Maurici da Silva. E-mail: rosemaurici@gmail.com

\section{Introdução}

O uso da ventilação mecânica (VM) aumentou nas últimas décadas ${ }^{1,2}$ e atualmente encontra-se entre as mais importantes modalidades terapêuticas em uma unidade de terapia intensiva (UTI) ${ }^{3}$. Estima-se uma prevalência de $42 \%$ de pacientes submetidos a esse recurso no Brasil, e de 39\% nos demais países".

Contudo, a VM invasiva está associada a complicações, como pneumonia, disfunção diafragmática, polineuropatia do paciente crítico, dentre outras que podem aumentar a morbidade e mortalidade ${ }^{5-}$ ${ }^{7}$. Nesse sentido, torna-se fundamental reduzir o tempo no qual o paciente está sob VM, reestabelecendo a ventilação espontânea tão logo seja possível, tornando a identificação de estratégias que reduzam a sua duração uma prioridade ${ }^{8,9}$.

O desmame da VM pode ser definido como o processo de retirada abrupta ou gradual do suporte ventilatório, e representa $40-50 \%$ da duração total da ventilação ${ }^{10-14}$. A descontinuidade da VM deve ser realizada logo que o paciente consiga manter a via aérea protegida e ventilação espontânea adequada, porém em pacientes com falência respiratória aguda e em condições graves, esse processo pode apresentar dificuldades, especialmente em função de características relativas às doencas de base, tornando o desmame ventilatório mais demorado. A "decisão de extubar" pode levar a consequências importantes, tanto o atraso da extubação quanto a falha da extubação, que estão associados a uma maior duração da ventilação mecânica e aumento da mortalidade $^{10}$. A descontinuação prematura exerce forte estresse sobre os sistemas respiratório e cardiovascular ${ }^{9}$, enquanto atrasos desnecessários podem levar a hipoatrofia diafragmática ${ }^{15}$. Os dados existentes indicam que o passo mais importante no processo de desmame ventilatório para evitar o prolongamento desnecessário da VM é o reconhecimento do momento para desmamar e para extubar.

A incidência de extubação não planejada varia de 0,3 a $16 \%^{10}$. Na maioria dos casos (83\%), a extubação não planejada é executada pelo póprio paciente, enquanto $17 \%$ são acidentais ${ }^{10}$. Quase metade dos pacientes com auto-extubação durante o período de desmame não necessita de reintubação, sugerindo que muitos pacientes são mantidos em ventilação mecânica além do tempo necessário ${ }^{4,16}$. 
Aproximadamente 60-70\% dos pacientes exigirá um mínimo ou nenhum desmame do suporte ventilatório e serão extubados sem dificuldade após o primeiro teste de respiração espontânea $(\mathrm{TRE})^{4}$. Os demais podem ser classificados como desmame difícil, definido como até três tentativas de TRE e sete dias para conseguir sucesso no desmame da VM ou desmame prolongado, definido como exigindo mais de três tentativas de TRE e mais de sete dias de desmame".

Foi comprovado que o aumento do tempo entre o dia do desmame da ventilação e da extubação efetiva aumenta significativamente a mortalidade ${ }^{4}$, assim como a reintubação por falha da extubação mostrou aumentar a mortalidade de 2,5 a 10 vezes em comparação com pacientes que não necessitam de reintubação ${ }^{17}$. Por isso, a possibilidade de prever tanto a falha do desmame da ventilação quanto da extubação auxiliaria no desfecho do paciente crítico, ou seja, a identificação de fatores de risco para falha de extubação baseado em evidências científicas poderia trazer inúmeras vantagens em relação ao desmame conduzido de forma empírica.

\section{Objetivos}

Identificar os fatores associados à falha de extubação, buscando a caracterização de pacientes com risco do seu insucesso, e avaliar as implicações clínicas relacionadas com a necessidade de reintubação.

\section{Método}

Foi realizado um estudo de coorte, devidamente aprovado pelo Comitê de Ética em Pesquisa em Seres Humanos da Universidade Federal de Santa Catarina sob o protocolo CAAE 42934615.3.0000.0118. Para cada paciente elegível, um termo de consentimento foi entregue a um familiar responsável e a inclusão somente ocorria mediante assinatura do mesmo.

Foram coletados dados de pacientes críticos internados na UTI adulto do Hospital Universitário Professor Polydoro Ernani de São Thiago da Universidade Federal de Santa Catarina (HU-UFSC) durante o período de junho de 2015 a maio de 2017. Incluiu-se todos os pacientes com idade igual ou superior a 18 anos de idade, intubados e submetidos à VM invasiva por pelo menos 48 horas ou mais, cujos responsáveis concordassem com a participação no estudo. Os pacientes inclusos foram acompanhados até 48 horas após a extubação, permanecendo aqueles que apresentassem o desfecho considerado neste estudo, como reintubação dentro de 48 horas após a extubação ou a evolução para o óbito durante esse período. A indicação de extubação foi avaliada pelo médico assistente

Critérios de exclusão abrangiam os pacientes cujos responsáveis não concordassem com a participação, pacientes que não foram submetidos ao processo de extubação devido a evolução para o óbito ou a realização imediata de traqueostomia, e naqueles que tivessem falta nos registros clínicos de qualquer informação relevante.

Nos pacientes que preencheram os critérios de inclusão, a equipe de pesquisa preencheu formulários de coleta de dados por meio de extração dos dados demográficos e clínicos, a partir do registro clínicos. As variáveis analisadas foram sexo, idade, índice de massa corporal, motivo da internação na UTI, causa da intubação, comorbidades e SAPS III (New Simplified Acute Physiology Score III) e APACHE II (Acute Physiology and Chronic Health Evaluation II) relativos à admissão na UTI. Também foram coletados dados do paramêtro ventilatório, valores da gasometria arterial e aspecto da secreção traqueal. Implicações clínicas associadas à VM também foram analisadas: tempo de VM, tempo de permanência na UTI, óbito na UTI ou não, pneumonia associada à VM, frequência de traqueostomia e necessidade de VNI pós-extubação. O desfecho primário foi falha de extubação, definida neste estudo como a necessidade de reintubação dentro de 48 horas após a extubação.

A análise dos dados foi realizada com o auxílio do software SPSS 22.0. Variáveis nominais foram sumarizadas como números absolutos e percentuais, e variáveis numéricas como média e desvio padrão quando de distribuição normal e mediana, valores máximos e mínimos quando de distribuição não normal. A normalidade dos dados foi avaliada pelo teste de Shapiro Wilk. A associação entre as variáveis de interesse com o desfecho foi avaliada por meio do teste de qui quadrado, $t$ de student ou ANOVA conforme apropriado. Correlações entre variáveis numéricas contínuas foram avaliadas por meio do coeficiente de correlação de Pearson ou Spearman. Foi adotado um nível de significância de $5 \%$.

\section{Resultados}

No período da coleta de dados, 144 pacientes foram triados quanto à potencial elegibilidade. Desse total, 61 foram excluídos. Assim, a análise final incluiu 83 pacientes. Destes, quando submetidos à extubação, $12(14,5 \%)$ apresentaram falha de extubação. (Figura 1) 


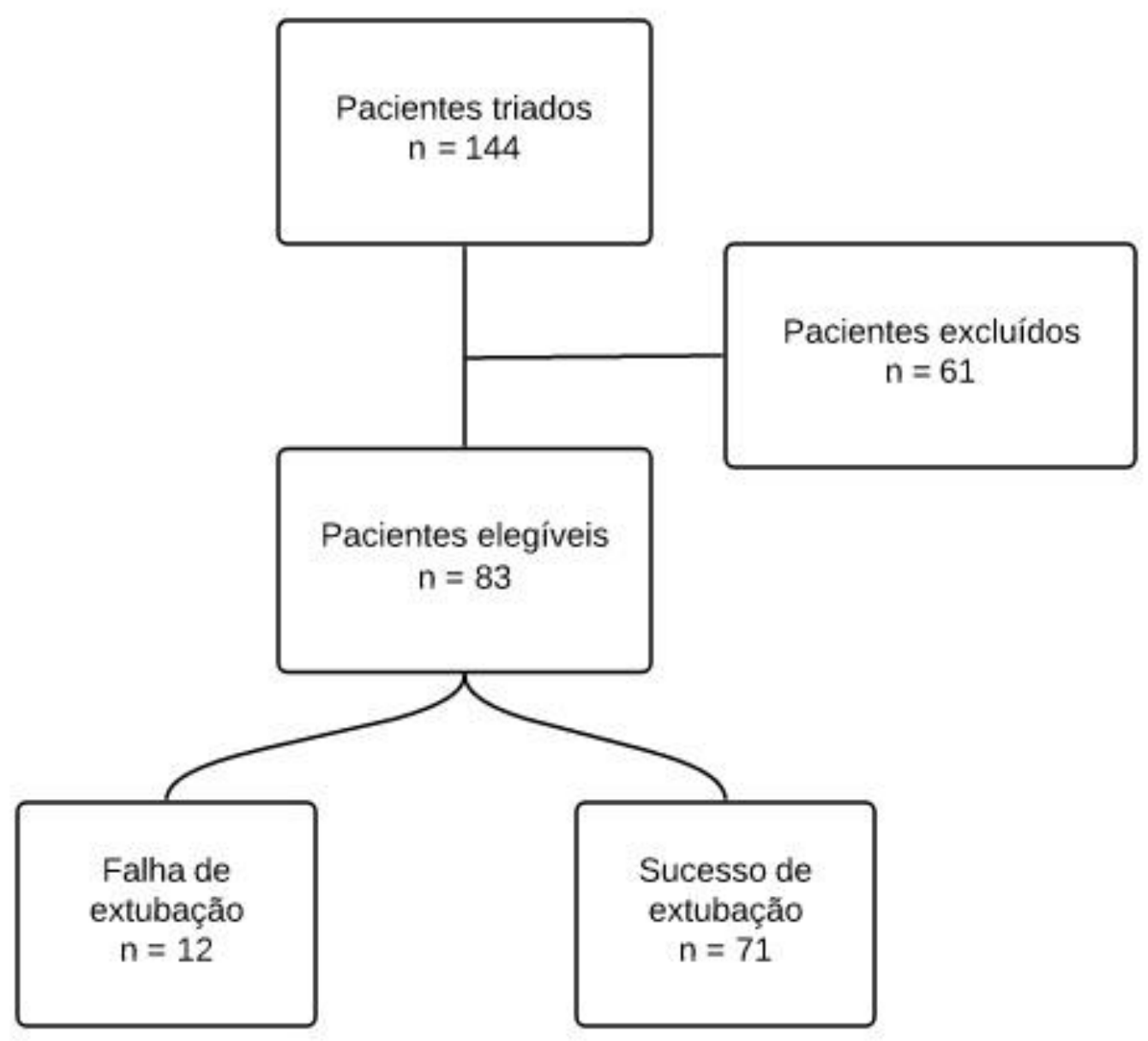

Figura 1. Fluxograma do estudo

A amostra de pacientes analisados mostrou discreto predomínio do sexo masculino $(55,4 \%)$, com uma maioria apresentando a faixa etária menor de 60 anos (53\%). O tempo médio de permanência na UTI e na VM foram de oito dias. (Tabela 1 e Tabela 2).

As principais causas que levaram estes pacientes à internação nesta UTI foram respectivamente em ordem decrescente: sepse $(28,9 \%)$, distúrbio respiratório $(18,1 \%)$ e pós-operatório $(26,5 \%)$. Na maioria dos casos, a falência respiratória $(42,2 \%)$ e a anestesia geral $(26,5 \%)$ foram os motivos que levaram esses pacientes a serem submetidos à intubação orotraqueal e manutenção em VM. Apesar das características da amostra, o diagnóstico inicial de sepse como indicação para internação na UTI não foi encontrado entre os pacientes que falharam após o processo de extubação, estes apresentaram como principais causas para necessidade de cuidados intensivos: pós-operatório $(41,7 \%)$ e distúrbios neurológicos (25\%). Os motivos que levaram à necessidade de suporte ventilatório entre os pacientes que foram reintubados permaneceram semelhantes em relação à amostra analisada (Tabela 3 e 4).

Tabela 1. Características da amostra estudada

\begin{tabular}{cc}
\hline & Média + desvio padrão \\
\hline Idade (anos) & $57 \pm 16$ \\
Índice de massa corporal & $26 \pm 6$ \\
SAPS III & $67 \pm 14$ \\
APACHE II & $20 \pm 7$ \\
Tempo UTI (dias) & $8 \pm 5$ \\
Tempo VM (dias) & $8 \pm 5$ \\
Volume corrente (ml) & $513 \pm 128$ \\
PEEP (cm de $\left.\mathrm{H}_{2} \mathrm{O}\right)$ & $7 \pm 1$ \\
$\mathrm{PaO}_{2}$ & $130 \pm 33$ \\
$\mathrm{PaO}_{2} / \mathrm{FiO}_{2}$ & $349 \pm 104$ \\
$\mathrm{PaCO}_{2}$ & $40 \pm 8$ \\
\hline
\end{tabular}


Tabela 2. Características de base entre os grupos de sucesso e falha de extubação

\begin{tabular}{|c|c|c|c|}
\hline Variáveis & $\begin{array}{c}\text { Sucesso } \\
(\mathrm{n}=71) \\
\mathrm{n}(\%)\end{array}$ & $\begin{array}{c}\text { Falha } \\
(\mathrm{n}=12) \\
\mathrm{n}(\%)\end{array}$ & $\mathrm{p}$ \\
\hline \multicolumn{3}{|l|}{ Sexo } & 0,826 \\
\hline Masculino & $39(54,9)$ & $7(58,3)$ & \\
\hline Feminino & $32(45,1)$ & $5(41,7)$ & \\
\hline \multicolumn{3}{|l|}{ Idade } & 0,395 \\
\hline Menor 60 anos & $39(54,9)$ & $5(41,7)$ & \\
\hline Maior 60 anos & $32(45,1)$ & $7(58,3)$ & \\
\hline IMC & 26 & 25 & 0,997 \\
\hline SAPS & 63 & 66 & 0,654 \\
\hline APACHE & 19 & 40 & 0,931 \\
\hline
\end{tabular}

Tabela 3. Causa de internação na UTI entre os grupos de sucesso e falha de extubação

\begin{tabular}{ccccc}
\hline Variáveis & Total $\mathrm{n}=83$ & $\begin{array}{c}\text { Sucesso } \\
\mathrm{n}=71(\%)\end{array}$ & $\begin{array}{c}\text { Falha } \\
\mathrm{n}=12(\%)\end{array}$ & $\mathrm{p}$ \\
\hline Sepse & & $24(33,8)$ & - & 0,017 \\
Distúrbio cardiovascular & 24 & $4(5,6)$ & $1(8,3)$ & 0,716 \\
Distúrbio respiratório & 5 & $13(18,3)$ & $2(16,7)$ & 0,891 \\
Distúrbio neurológico & 5 & $8(11,3)$ & $3(25,0)$ & 0,194 \\
Distúrbio gastrointestinal & 11 & $4(5,6)$ & $2(16,7)$ & 0,172 \\
Pós-operatório & 6 & $17(23,9)$ & $5(41,7)$ & 0,198 \\
Outro & 22 & $1(1,4)$ & - & 0,665 \\
\hline
\end{tabular}

Tabela 4. Causa de insuficiência respiratória entre os grupos de sucesso e falha de extubação

\begin{tabular}{ccccl}
\hline Variáveis & Total & Sucesso & Falha & Valor \\
$\mathrm{n}=83$ & $\mathrm{n}=71(\%)$ & $\mathrm{n}=12(\%)$ & 0 \\
\hline Falência respiratória & 35 & $31(43,7)$ & $4(33,3)$ & 0,503 \\
Falência hemodinâmica & 13 & $12(16,9)$ & $1(8,3)$ & 0,450 \\
Falência neurológica & 13 & $10(14,1)$ & $3(25,0)$ & 0,336 \\
Anestesia Geral & 22 & $18(25,4)$ & $4(33,3)$ & 0,562 \\
\hline
\end{tabular}

No presente estudo a amostra apresentou bastante heterogênea tanto no grupo de falha quanto ao grupo de sucesso de extubação. (Tabela 4). A maioria dos indivíduos apresentou média quantidade de secreção traqueal com aspecto purulento, contudo as características da secreção traqueal não apresentaram associação com falha de extubação. Também não foram observadas associações significantes entre os dados gasométricos e os parâmetros ventilatórios, com a necessidade de reintubação. (Tabelas 5 e 6).

Tabela 5. Comorbidades entre os grupos de sucesso e falha de extubação

\begin{tabular}{ccccc}
\hline Variáveis & $\begin{array}{c}\text { Total } \\
\mathrm{n}=83\end{array}$ & $\begin{array}{c}\text { Sucesso } \\
\mathrm{n}=71(\%)\end{array}$ & $\begin{array}{c}\text { Falha } \\
\mathrm{n}=12(\%)\end{array}$ & $\mathrm{p}$ \\
\hline DPOC & 5 & $4(5,6)$ & $1(8,3)$ & 0,726 \\
Insuficiência Cardíaca & 2 & $2(2,8)$ & - & 0,556 \\
Cirrose & 6 & $5(7,0)$ & $1(8,3)$ & 0,873 \\
Neoplasia & 9 & $7(9,9)$ & $2(16,7)$ & 0,495 \\
Insuficiência Renal Aguda & 6 & $5(7,0)$ & $1(8,3)$ & 0,873 \\
Aids & 2 & $2(2,8)$ & - & 0,556 \\
Outros & 43 & $37(52,1)$ & $6(50,0)$ & 0,892 \\
\hline
\end{tabular}


Tabela 6. Parâmetros gasométricos e ventilatórios entre os grupos de sucesso e falha de extubação

\begin{tabular}{ccccc}
\hline Variáveis & $\begin{array}{c}\text { Total } \\
(\mathrm{n}=83)\end{array}$ & $\begin{array}{c}\text { Sucesso } \\
(\mathrm{n}=71)\end{array}$ & $\begin{array}{c}\text { Falha } \\
(\mathrm{n}=12)\end{array}$ & $\mathrm{p}$ \\
\hline Volume Corrente $(\mathrm{ml})$ & $513 \pm 128$ & 515 & 493 & 0,423 \\
$\mathrm{PEEP}$ & $7 \pm 1$ & 7 & 7 & 0,470 \\
$\mathrm{PaO}_{2}(\mathrm{mmHg})$ & $130 \pm 33$ & 132,6 & 119 & 0,373 \\
$\mathrm{PaCO}_{2}(\mathrm{mmHg})$ & $349 \pm 104$ & 39,6 & 42,1 & 0,470 \\
$\mathrm{PaO}_{2} / \mathrm{FiO}_{2}$ & $40 \pm 8$ & 356,6 & 303,4 & 0,130 \\
$\mathrm{SatO}_{2}(\%)$ & $98 \pm 1,6$ & $98 \%$ & 97 & 0,703 \\
\hline
\end{tabular}

Tabela 7. Características da secreção traqueal entre os grupos de sucesso e falha de extubação

\begin{tabular}{ccccc}
\hline Variáveis & Total & Sucesso & Falha & $\mathrm{p}$ \\
& $\mathrm{n}=83$ & $\mathrm{n}=71(\%)$ & $\mathrm{n}=12(\%)$ & \\
\hline Pequena quantidade purulenta & 19 & $18(25,4)$ & $1(8,3)$ & 0,187 \\
Pequena quantidade hialina & 7 & $6(8,5)$ & $1(8,3)$ & 0,978 \\
Média quantidade purulenta & 41 & $33(46,5)$ & $8(66,7)$ & 0,211 \\
Média quantidade hialina & 3 & $3(4,2)$ & - & 0,465 \\
Grande quantidade purulenta & 10 & $8(11,3)$ & $2(16,7)$ & 0,608 \\
Grande quantidade hialina & 2 & $2(2,8)$ & - & 0,553 \\
\hline
\end{tabular}

Houve associação significante entre o maior tempo de UTI (p 0,048), maior tempo de VM (p 0,040) e mortalidade na UTI (p 0,022) com falha de extubação. Foram também observadas maior número de complicações associadas ao uso do suporte ventilatório, como pneumonia associada à $\mathrm{VM}(\mathrm{p} 0,001)$ e frequência de traqueostomia (p 0,009). (Tabela 8)

Tabela 8. Implicações clínicas entre os grupos sucesso e falha de extubação

\begin{tabular}{ccccc}
\hline Variáveis & $\begin{array}{c}\text { Total } \\
(\mathrm{n}=83)\end{array}$ & $\begin{array}{c}\text { Sucesso } \\
(\mathrm{n}=71)\end{array}$ & $\begin{array}{c}\text { Falha } \\
(\mathrm{n}=12)\end{array}$ & $\begin{array}{c}\text { Valor } \\
\text { de } \mathrm{p}\end{array}$ \\
\hline Mortalidade na UTI & 9 & 5 & 4 & 0,022 \\
Mortalidade hospitalar & 19 & 15 & 4 & 0,352 \\
Tempo de VM (dias) & $8 \pm 5$ & 7 & 24 & 0,040 \\
Tempo de internação na UTI (dias) & $8 \pm 5$ & 38 & 14 & 0,048 \\
Pneumonia associada à VM & 8 & 3 & 5 & 0,001 \\
Traqueostomia & 16 & 10 & 6 & 0,009 \\
VNI pós-extubação & 40 & 37 & 3 & 0,082 \\
\hline
\end{tabular}

\section{Discussão}

No presente estudo, não foram encontradas variáveis que apresentassem associação significante com falha de extubação, não conseguindo caracterizar quais seriam os pacientes que desenvolveriam maior risco de reintubação dentro das primeiras 48 horas. $\mathrm{O}$ impacto da falha de extubação foi significativa, associando-se com aumento da mortalidade na UTI, maior permanência no suporte ventilatório e internação na UTI, e à complicações relacionadas a uso da VM prolongada, como traqueostomia e pneumonia.

A incidência de falha de extubação foi de 14,5\%. A incidência de falha na retirada do suporte ventilatório encontrada na literatura varia de 5 a $20 \%{ }^{12,141,1,19}$, sendo que uma interessante revisão que pretendia responder "qual a taxa ideal de falha de extubação?”"19 através da média entre estudos observacionais e intervencionais encontrou uma taxa de $14 \%$, e que valores acima disso são inapropriadamente elevados. Este estudo apresentou taxa de insucesso da desvinculação da VM semelhante ao encontrado na literatura e não está tão distante da taxa ideal apontada pela revisão citada. Apesar de não estar efetivamente implementado um protocolo de desmame da ventilação, este fato é compensado por rounds multidisciplinares diários, nos quais cada paciente é individualizado e são revisados desmame da sedoanalgesia e possibilidade de desmame do suporte ventilatório acompanhado de seus fatores de risco e o atendimento fisioterápico contínuo, já que é sabido que a reabilitação física na UTI, quando iniciada em um ou dois dias após o início da VM, é viável, bem tolerada e benéfica, incluindo melhorias na capacidade física, estado funcional na alta hospitalar, diminuição do tempo em VM, e menor tempo de permanência na UTI ${ }^{20}$.

Vale lembrar que uma taxa muito baixa de reintubação (menores que $10 \%$ ) provavelmente demonstra pouco atrevimento no momento da decisão de extubar. Além disso, se a taxa de reintubação de um 
médico for próxima de zero, os pacientes podem de fato estar expostos a um risco maior de lesão pulmonar associada à VM, já que provavelmente está se retardando o momento de extubação ${ }^{21}$.

A amostra deste estudo evidenciou uma predominância do sexo masculino $(55,4 \%)$ no grupo de falha de extubação, contudo, não foi encontrada diferença significante relacionada à variável, o que confirma os achados de outros estudos ${ }^{22-24}$. Quanto à faixa etária, houve maior frequência de falha de extubação na população acima de 60 anos, contudo, não foi observada associação entre falha de extubação e a idade como foi observado nos estudos de Thille et al. ${ }^{18}$ e Frutos Vivar et al. ${ }^{22}$. Isso pode ser explicado pela característica do local onde se encontra a UTI, uma cidade turística com predomínio de uma população adulta $(12 \%$ da população da capital catarinense ocupa a faixa etária entre 20-59 anos segundo dados do IBGE 2010) a qual aumenta durante o período de maior incidência de turista. Esse dado explica a idade média da amostra deste trabalho apresentar-se como 57 anos.

Não foram encontradas associações entre o motivo da necessidade de internação na UTI com falha de extubação. Porém não há como não notar que nenhum paciente com quadro clínico de sepse que necessitou de internação na UTI e VM apresentou falha de extubação, mesmo sendo a maioria da população deste estudo. Diferente deste achado, um estudo mostrou que pacientes sépticos tendem a apresentar falha no desmame da ventilação no primeiro $\operatorname{dia}^{25}$. Já se sabe que a sepse provoca miopatia caracterizada pela redução da capacidade de geração de força muscular, e atrofia em 70 a $100 \%$ dos $\operatorname{casos}^{26}$. Cientes disso, talvez neste grupo de pacientes, os médicos intensivistas foram zelosos na indicação para extubação, diferindo os critérios em relação aos pacientes com necessidade de cuidados pós-operatórios.

Causa da falência respiratória e comorbidades não se correlacionaram de forma significativa com falha de extubação no presente estudo. Contudo, alguns autores observaram que pneumonia como causa do início do suporte ventilatório invasivo e comorbidades como insuficiência cardíaca, doenças respiratórias crônicas e doenças neurológicas foram fatores significativamente associados à ocorrência de falha de extubação $^{27,28,18,22}$, o que pode não ter sido identificado nessa pesquisa devido à pequena amostra incluída ou perfil clínico dos indivíduos estudados.

No trabalho de Mokhlesi $i^{24}$, a $\mathrm{PaCO}_{2} \geq 44 \mathrm{mmHg}$ foi um preditor sensível de falha de extubação. A reintubação provavelmente ocorre quando há um desequilíbrio entre a carga imposta aos músculos respiratórios e a capacidade de resposta dos músculos, levando à hipoventilação alveolar. Nesta pesquisa, não foi encontrada associação deste dado gasométrico com a falha de extubação, devido à padronização da coleta de dados da gasometria num horário fixo e assim, não há dados da $\mathrm{PaCO}_{2}$ no momento do TRE.
Neste trabalho não foi encontrada associação de falha de extubação e a quantidade e o aspecto da secreção traqueal. Novamente citando o estudo de Mokhlesi ${ }^{24}$, a quantidade de secreção em vias aéreas foi um dos fatores considerados como preditores de falha de extubação. Observaram que pacientes com secreção moderada ou com necessidade de aspiração a cada 1 a 2 horas ou diversas vezes por hora apresentaram maior probabilidade de evoluírem com necessidade de reintubação. Khamies et al. ${ }^{29}$ identificaram fatores de "competência nas vias aéreas", ou seja, tosse eficaz e quantidade de secreção traqueal, como preditores importantes do resultado da extubação em uma coorte de pacientes que já concluíram com sucesso o TRE. A divergência dos achados provavelmente aconteceu devido já ser sabido pela equipe multidisciplinar que volumosas quantidades de secreção traqueal assim como necessidade frequente de aspirações da via aérea estão associadas à falha, levando assim a um atraso da extubação. Associado a este fato, não possuímos dados referente à avaliação da eficácia da tosse nesta amostra, o qual tornaria mais completa a avaliação da capacidade de manter patente a via área, por exemplo, o paciente poderia apresentar moderada quantidade de secreção, contudo apresentava tosse eficaz reduzindo as chances de falha de extubação.

Não foi encontrada na análise dos dados a associação da ventilação mecânica não invasiva (VNI) com falha de extubação. Contudo, se faz necessária à distinção do uso da VNI como medida profilática para evitar um dano respiratório agudo ou como terapêutica para a insuficiência respiratória pós-extubação, justificando o achado deste trabalho. Os estudos apontam que o uso da VNI como possível estratégia para profilaxia da insuficiência respiratória pós-extubação em pacientes de alto risco pode ser útil. Uma meta-análise ${ }^{30}$ composta por quatro estudos mostrou que a VNI, comparada com a terapêutica médica, não diminuiu a reintubação ou mortalidade na UTI em pacientes com insuficiência respiratória pós-extubação. No entanto, em pacientes que foram definidos como com alto risco de desenvolver este distúrbio respiratório, a VNI conseguiu impactar nas taxas de reintubação e na mortalidade dentro da UTI. Contudo, quando a VNI é empregada como medida terapêutica não há benefício comprovado ${ }^{31}$ e pode até aumentar a mortalidade atrasando a reintubação ${ }^{32}$.

O impacto da reintubação nesses pacientes assemelha-se aos achados de outros estudos: a falha de extubação está associada a aumento da mortalidade na UTI e do seu tempo de permanência e duração na ventilação mecânica ${ }^{10,11,14}$, logo, aumenta os riscos de complicações associadas a esses fatores, notadamente a pneumonia associada à VM e a traqueostomia. Uma metanálise ${ }^{20}$ avaliou os resultados obtidos em 16 estudos, e indicou que a reintubação é um fator de risco para pneumonia associada à VM.

Este trabalho possui algumas limitações como o tamanho pequeno da amostra e a coleta de dados se 
restringirem a um único centro. Além disso, fatores de risco já relatados na literatura, como por exemplo, quantidade abundante de secreção e tosse ineficaz (mesmo que não analisado este dado), já estão incorporados na rotina da equipe da UTI no momento do desmame da VM, por isso dificilmente iriam mostrar associação significante neste estudo. Também não foram considerados dados de possíveis preditores para extubação como a avaliação de tosse, teste de cuff leak, balanço hídrico, a saturação venosa central, índice de respiração rápida e superficial e índice de Tobin. As decisões de extubação foram definidas pelo médico assistente presente no momento da extubação, de acordo com seus conhecimentos e experiência clínica, assim a decisão da retirada do suporte ventilatório não seguiu um protocolo de desmame da ventilação.

\section{Conclusão}

A falha de extubação está associada a aumento da mortalidade na UTI, do tempo de permanência, e duração na ventilação mecânica.

\section{Referências}

1. Braun SR, Smith RF, McCarthy TM, Minsloff M. Evaluating the changing role of respiratory therapy services at two hospitals. JAMA 1981;245:2033-37.

2. Snider GL. Historical perspective on mechanical ventilation: from simple life support system to ethical dilemma. Am Rev Respir Dis 1989;140:52-7.

3. Esteban A, Alia I, Ibanez J, Benito S, Tobin MJ. Modes of mechanical ventilation and weaning. A national survey of Spanish hospitals. The Spanish Lung Failure

Collaborative Group. Chest 1994;106:1188-93.

4. Boles JM, Bion J, Connors A, Herridge M, Marsh B, Melot C, et al. Weaning from mechanical ventilation:. Eur Respir J 2007;29:1033-56.

5. Adamides AA, Cooper DJ, Rosenfeldt FL, Bailey MJ, Pratt N, Tippett N, et al. Focal cerebral oxygenation and neurological outcome with or without brain tissue oxygen-guided therapy in patients with traumatic brain injury. Acta Neurochir 2009;151:1399-409.

6. MacIntyre NR, Cook DJ, Ely EW Jr, Epstein SK, Fink JB, et al. American College of Chest Physicians; American Association for Respiratory Care; American College of Critical Care Medicine: Evidence-based guidelines for weaning and discontinuing ventilatory support: A collective task force facilitated by the American College of Chest Physicians; the American Association for Respiratory Care; and the American College of Critical Care Medicine. Chest 2001;120(Suppl 6):375S$395 \mathrm{~S}$.

7. Esteban A, Frutos F, Tobin MJ, Alía I, Solsona JF, Valverdú I, et al. A comparison of four methods of weaning patients from mechanical ventilation. N Engl J Med 1995;332:345-50.

8. Brochard L, Rauss A, Benito S, Conti G, Mancebo J, Rekik N, et al. Comparison of three methods of gradual withdrawal from ventilatory support during weaning from mechanical ventilation. Am J Respir Crit Care Med 1994;150:896-903.

9. Girard TD, Ely WE. Protocol-driven ventilator weaning: Reviewing the evidence. Clin Chest Med 2008;29:241-252.

10. Epstein SK. Decision to extubate. Intensive Care Med 2002;28:535-46.

11. Esteban A, Anzueto A, Frutos F, Alía I, Brochard L, Stewart TE, et al. Mechanical Ventilation International Study Group. Characteristics and outcomes in adult patients receiving mechanical ventilation: a 28-day international study. JAMA 2002;287:345-55.

12. Esteban A, Alia I, Ibanez J, Benito S, Tobin MJ. Modes of mechanical ventilation and weaning. A national survey of Spanish hospitals. The Spanish Lung Failure Collaborative Group. Chest 1994; 106:1188-93.

13. Ely EW, Baker AM, Dunagan DP, Burke HL, Smith AC, Kelly PT, et al. Effect on the duration of mechanical ventilation of identifying patients capable of breathing spontaneously. N Engl J Med 1996;335:1864-69.

14. Kollef MH, Shapiro SD, Silver P, St John RE, Prentice D, Sauer S, et al. A randomized, controlled trial of protocol-directed versus physician directed weaning from mechanical ventilation. Crit Care Med 1997;25:567-74.

15. Levine S, Nguyen T, Taylor N, Friscia ME, Budak MT, Rothenberg P, et al. Rapid disuse atrophy of diaphragm fibers in mechanically ventilated humans. N Engl J Med 2008, 358:1327-35.

16. Epstein SK, Nevins ML, Chung J. Effect of unplanned extubation on outcome of mechanical ventilation. Am J Respir Crit Care Med 2000;161:1912-16.

17. Rothaar RC, Epstein SK. Extubation failure: magnitude of the problem, impact on outcomes, and prevention. Curr Opin Crit Care 2003;9:59-66.

18. Frutos-Vivar F, Esteban A, Apeztequia C, González M, Arabi Y, et al. Outcome of reintubated patients after scheduled extubation. J Crit Care 2011;26:502-9.

19. Macintyre N. Discontinuing Mechanical Ventilatory Support. Chest 2007;132(3):1049-56.

20. Rothaar RC, Epstein SK. Extubation failure: magnitude of the problem, impact on outcomes, and prevention. Curr Opin Crit Care 2003;9:59-66.

21. Chen L, Gilstrap D, Cox CE. Mechanical Ventilator Discontinuation Process. Clin Chest Med. 2016 Dec;37(4):693-699.

22. Thille AW, Harrois A, Schortgen F, Brun-Buisson C, Brochard L. Outcomes of extubation failure in medical intensive care unit patients. Crit Care Med 2011;39:2612-2618.

23. Penuelas O, Frutos-Vivar F, Fernandez C, Anzueto A, Epstein SK, Apezteguía C, et al. Characteristics and outcomes of ventilated patients according to time to liberation from mechanical ventilation. Am J Respir Crit Care Med 2011; 184:430-437.

24. Mokhlesi B, Tulaimat A, Gluckman TJ, Wang Y, Evans AT, Corbridge TC. Predicting extubation failure after successful completion of a spontaneous breathing trial. Respir Care 2007;52(12):1710-7.

25. Amoateng-Adjepong Y, Jacob BK, Ahmad M, Manthous CA. The Effect of Sepsis on Breathing Pattern and Weaning Outcomes in Patients Recovering From Respiratory Failure. Chest 1997;112(2): 472-77.

26. Callahan LA, Supinski GS. Sepsis-induced myopathy. Crit Care Med 2009;37(10 Suppl):S354-67.

27. Vallverdu I, Calaf N, Subirana M, Net A, Benito S, Mancebo J. Clinical characteristics, respiratory functional parameters, and outcome of a two-hour T-piece trial in patients weaning from mechanical ventilation. Am J Respir Crit Care Med 1998;158:1855-62.

28. Epstein SK, Ciubotaru RL. Independent effect of etiology of failure and time to reintubation on outcome for patients failing extubation. Am J Respir Crit Care Med 1998;158:489-93.

29. Khamiees M, Raju P, DeGirolamo A, Amoateng-Adjepong Y, Manthous CA. Predictors of extubation outcome in patients who have successfully completed a spontaneous breathing trial. Chest 2001;120:1262-70.

30. Agarwal R, Aggarwal AN, Gupta D, Jindal SK. Role of noninvasive positivepressure ventilation in postextubation respiratory failure: a meta-analysis. Respir Care 2007;52:1472-79.

31. Keenan SP, Powers C, McCormack DG, Block G. Noninvasive positive-pressure ventilation for postextubation respiratory distress: a randomized controlled trial. JAMA 2002;287:3238-44.

32. Esteban A, Frutos-Vivar F, Ferguson ND, Yasseen A, Apezteguía C, González M, et al. Noninvasive positive-pressure ventilation for respiratory failure after extubation. N Engl J Med 2004;350:2452-60. 